\title{
Enfrentando a COVID-19: APS forte agora mais que nunca!
}

\author{
Fúlvio Borges Nedel
}

\section{RESUMO}

Este artigo apresenta uma breve reflexão sobre os desafios da Atenção Básica do SUS diante da epidemia de COVID-19 e de como eles impactam os princípios e atributos da Atenção Primária em Saúde (APS), além de propor ações para este momento. As demandas trazidas pela epidemia, ainda que requeiram métodos de intervenção inovadores, exigem a reafirmação de princípios fundadores da APS, como a universalização e o trabalho em equipe generalista com prática clínica e comunitária organizada sobre uma base territorial, e sugerem a revisão da ideia de "orientação comunitária" como um "atributo derivado". Propõe-se como uma das estratégias de enfrentamento da COVID-19 a universalização imediata da Estratégia Saúde da Família, com a contratação emergencial de equipes de SF e revitalização dos Núcleos de Apoio à Saúde da Família.

Palavras-chave: Pandemia, COVID-19, Atenção Primária à Saúde; Saúde da Família; Saúde Comunitária; Sistemas de Saúde.

Revista da Rede APS 2020

Publicada em: 15/04/2020

DOI:10.14295/aps.v2i1.68

Fúlvio Borges Nedel (Departamento de Saúde Pública da Universidade Federal de Santa Catarina, Santa Carina, Brasil);

Correspondência para: Fúlvio Borges Nedel fulvionedel@gmail.com

\section{ABSTRACT}

This article presents a brief reflection on the challenges of SUS Primary Care in the face of the COVID-19 epidemic and how they impact the principles and attributes of Primary Health Care (PHC). The demands brought about by the epidemic, even though they require innovative methods of intervention, require the reaffirmation of PHC founding principles, such as universalization and generalist teamwork with clinical and community practice organized on a territorial basis, and suggest a review of the idea of "Community orientation" as a "derived attribute". As one of the coping strategies of COVID-19, the immediate universalization of the Family Health Strategy is proposed, with the emergency hiring of FH teams and revitalization of the Family Health Support Centers.

Keywords: Pandemic, COVID-19, Primary Health Care; Family Health; Community health; Health Systems. 
Nedel, F. B.

\section{INTRODUÇÃO}

A pandemia da COVID-19 abre o ano de 2020 como uma notícia alarmante para uns poucos então chamados alarmistas e antes de que terminasse o trimestre já era uma das principais, senão a principal notícia na grande imprensa e na mídia alternativa, além das tele-conversas com amigxs e familiares. Os que podemos, estamos em casa. Os que podemos trabalhar de casa ou não podem ir ao trabalho porque este está suspenso, temos o dever de não sair de casa, porque é a grande diminuição de circulação de pessoas o que pode evitar as mortes que se esperam ${ }^{1,2}$. Não os necessários leitos de UTI que já existem ou se constroem, tampouco as máscaras caseiras para a população se ela sair à rua, como para ir trabalhar ou para pagar as contas ou retirar dinheiro. O que pode realmente diminuir as mortes esperadas por COVID-19 no Brasil, assim como em outras partes do mundo, é o isolamento social com a contenção das pessoas em seus domicílios e a identificação de casos. ${ }^{3,4,5}$

As manifestações clínicas da doença, por outro lado, são leves ou inexistentes na maioria dos infectados, enquanto uma pequena proporção de casos, que pode ser expressiva em alguns "grupos de risco", tem sintomatologia severa, que exige a hospitalização e muitas vezes cuidados intensivos $^{6,7}$. Na mídia, vemos que o sistema de saúde está presente em ações de gestão, vigilância e atenção hospitalar, seja ambulatorial pré-hospitalar, intra-hospitalar não intensivo ou de cuidados intensivos, mas pouco se vê sobre o primeiro nível de atenção e o mesmo se vê em publicações acadêmicas, seja em análises atuais ou em previsões do problema que agora vivemos ${ }^{8}$. Na Inglaterra, a APS é orientada a atender à distância os casos suspeitos ou confirmados. Na Espanha, Centros de Atenção Primária foram fechados e suas equipes trasladadas a hospitais, dificultando o acesso para outros problemas, exaurindo e desorganizando a APS, que agora deve atender à "avalanche de altas hospitalares" e de pacientes críticos em tratamento domiciliar ${ }^{9}$.
Entretanto, no Reino Unido percebe-se a importância da APS para a identificação de casos através da testagem sorológica ${ }^{10}$, e se propõe, para promover o isolamento social, a contratação emergencial de Agentes Comunitários de Saúde (ACS), inclusive citando o sucesso dessa estratégia no Brasil ${ }^{11}$.

Assim, vale perguntar qual o papel do chamado "primeiro nível de atenção" dos sistemas de saúde, particularmente da $A B$ do SUS diante da epidemia. Em que as novas demandas exigem ações inovadoras, essas inovações estão além dos princípios e atributos da APS, ou prescindem deles, exigindo uma nova concepção teórica sobre a organização da primeira instância de atenção nos sistemas de saúde? Isto é, a epidemia representa uma quebra de paradigmas na Atenção Primária em Saúde (APS)?

\section{QUAL O PAPEL DA ATENÇÃo BÁsICA dO} SUS?

Não vale a pena, num texto curto, buscar um pífio papel que eventualmente pudesse ter, para o controle da epidemia, a primeira instância de atenção do setor privado (frequentemente realizada por especialistas, mas efetivamente utilizada como primeira instância de atenção em saúde). Mas sim vale notar que parece ser consenso que só o Estado pode resolver ou mesmo tratar do assunto. Não vemos, nos telejornais, um apelo ao Mercado para que, livre de amarras do Estado, resolva o problema e nos garanta a liberdade sonhada pelo neoliberalismo. É verdade que tampouco vemos na grande imprensa notícia das propostas de taxação de grandes fortunas ou das discussões sobre a necessidade de acabar como neoliberalismo, antes de que ele acabe com o mundo. Mas claramente a pandemia demonstra a importância de sistemas universais de saúde. ${ }^{12}$

A epidemia não diminui a demanda tradicional da $A B$, que deve agora atender tanto a população contaminada quanto a não contaminada, mas sem promover o contágio. A divisão de usuários em corredores de um 
Nedel, F. B.

mesmo ambiente não evita o contágio e a Associação Brasileira de Saúde Coletiva (ABRASCO), assim como a OMS, recomenda a montagem de tendas para a atenção dos casos suspeitos e diagnosticados de COVID, com equipes diferentes. Para ambas populações deve-se promover a atenção à distância, mas em ambos casos sempre haverá quem precise ir à Unidade de Atenção Primária, seja ela a sua conhecida Unidade Básica de Saúde, ou uma tenda provisória. Deve-se ainda lembrar a carga emocional sobre todos nós nesse momento e particularmente das pessoas suspeitas ou com diagnóstico da doença. Assim, é mais que nunca importante uma atenção integral e com forte vínculo. Essa é uma contribuição fundamental que $a B$ pode dar à população neste momento: continuar atendendo as pessoas, trabalhar em equipe, ainda que à distância, para garantir atenção mais integral. Garantir a continuidade da atenção, porque muitas pessoas voltarão dos hospitais e muitas pessoas estarão em luto ${ }^{13}$. Essas pessoas e famílias precisam e precisarão de atenção em saúde, uma atenção que é tipicamente da primeira instância (ou "nível", se ainda representássemos o sistema de saúde com uma pirâmide) e que será melhor se for realizada em equipe e pela equipe que já conhece.

Para a necessária ação de identificação de casos também é na $A B$ que pode estar a maior fortaleza do sistema, através da clínica individual e familiar e ações comunitárias. Mas além dessa, a $A B$ pode dar uma contribuição muito maior para o controle da epidemia, promovendo o isolamento social através de ações de saúde comunitária ${ }^{14}$. Não nos falta muito mais que relembrar as inciativas de saúde comunitária de poucos anos, ou mesmo os treinamentos de ACS ou de equipes de Saúde da Família (eqSF) do início da Estratégia Saúde da Família (ESF). As eqSF podem ser centros de concentração de repasse de informação de saúde e de Assistência Social, apoiada por Núcleos de Apoio à Saúde da Família (NASF), com grande papel para os $\mathrm{ACS}^{15}$. Outro exemplo, as eqSF podem organizar exemplo as compras de moradores, o que pode ser feito por ruas das micro-áreas, novamente tendo o ACS papel de destaque. Experiências e ideias em saúde comunitária não nos faltam, como temos visto em mostras de saúde promovidas pelo Ministério da Saúde e Organização Panamericana da Saúde.

A equipe terá então de desenvolver aptidões de telecomunicação, lembrando que há ainda muita gente sem telefone celular, ou fixo. No "Brasil remoto", em cidades de médio porte e também em comunidades das grandes cidades, essa comunicação terá de ser feita pela rádio ou outras formas, e a equipe terá então de recuperar aptidões esquecidas pela APS brasileira. Para a educação e vigilância em saúde, a "tele" comunicação do ACS na calçada com o morador em sua casa, a uma distância de pelo menos $2 \mathrm{~m}$, deve ser considerada e pode talvez aumentar o vínculo da equipe com os moradores.

\section{QUAL O MELHOR MODELO PARA A}

\section{PRIMEIRA INSTÂNCIA DE ATENÇÃO EM}

\section{SAÚDE?}

Parece que entre os aprendizados do momento está a necessidade de fortalecer o Sistema Único de Saúde e torná-lo efetivamente universal, baseado na APS. Tudo mostra a oportunidade das críticas aos retrocessos da Declaração de Astana, comparada com Alma-Ata15. Não parece haver uma quebra de paradigma, pelo contrário, os princípios fundadores da APS saem fortalecidos. Para dar conta de atender aos problemas típicos da $A B$ e para atender às demandas trazidas pela COVID-19, precisamos uma APS que recupere seus princípios de base comunitária, trabalho em equipe, intra e extramuros, com usuários, pacientes, famílias e comunidade. Que busque atenção integral e não cumprir uma carteira de serviços (que não previam a COVID-19). Que entenda que a integralidade depende do trabalho em equipe sobre um território, do qual não pode prescindir a clínica ampliada. Finalmente, parece que o atributo de "orientação comunitária" da APS 
Nedel, F. B.

não é "derivado", como propõe Starfield ${ }^{16}$, mas sim "essencial", inclusive para alcançar a integralidade.

\section{CONCLUSÃO}

É necessário assim um trabalho, talvez duplicado, de clínica individual e familiar e um trabalho comunitário, com muito uso de comunicação à distância em ambos casos. Precisamos inovar em alguns métodos e talvez recuperar outros antigos. Aumentar o acesso, porque pessoas fora do sistema de saúde são potenciais vetores desconhecidos. Fortalecer ainda mais o vínculo com as pessoas, e entender sua situação inserida em um "território vivo", através de sua configuração familiar e na comunidade em que vive. Isto é, necessitamos de uma $A B$ realmente universal e nos moldes da $E S F$, que se pode definir como APS, porque a $A B$ em seu modelo "Tradicional" (ambulatorial, de "especialidade geral", sem trabalho em equipe, exclusivamente intra-muros e sem base territorial) é incapaz de atender às demandas trazidas pela pandemia.

Essas necessidades são urgentes e tanto melhor se já tivessem sido atendidas. Necessitam recursos financeiros, humanos e materiais e a ação imediata e continuada do Estado. Assim, entre as primeiras ações de governo estão a revogação da Emenda Constitucional $n^{\circ} 95$, que estrangula o já subfinanciado SUS, e o aumento do seu financiamento, possivelmente através da taxação de grandes fortunas. O Brasil tem experiência recente na contratação de médicos, através do Programa Mais Médicos. Assim, propõe-se como uma das estratégias de enfrentamento da COVID-19 a universalização imediata da ESF, com a contratação emergencial de eqSF e a revitalização dos Núcleos de Apoio à Saúde da Família (NASF), que poderia ser feito através de um Programa Mais Saúde da Família. 


\section{NOTAS E REFERÊNCIAS}

1 Ferguson NM, Laydon D, Nedjati-Gilani G, Imai N, Ainslie K, Baguelin M, et al. Impact of nonpharmaceutical interventions (NPIs) to reduce COVID-19 mortality and healthcare demand. ImperialAcUk. 2020;

2 Remuzzi A, Remuzzi G. COVID-19 and Italy: what next? The Lancet. 2020

${ }^{3}$ Gt Walker P, Whittaker C, Watson O, Baguelin M, Ainslie KEC, Bhatia S, et al. The Global Impact of COVID19 and Strategies for Mitigation and Suppression. Imp Coll COVID-19 Response Team. 2020

4 Li R, Pei S, Chen B, Song Y, Zhang T, Yang W, et al. Substantial undocumented infection facilitates the rapid dissemination of novel coronavirus (SARS-CoV2). Science (80- ). 16 de março de 2020; eabb3221

${ }^{5}$ Fraher EP, Pittman P, Frogner BK, Spetz J, Moore J, Beck AJ, et al. Ensuring and Sustaining a Pandemic Workforce. N Engl J Med. 8 de abril de 2020; NEJMp2006376.

6 Driggin E, Madhavan M V., Bikdeli B, Chuich T, Laracy J, Bondi-Zoccai G, et al. Cardiovascular Considerations for Patients, Health Care Workers, and Health Systems During the Coronavirus Disease 2019 (COVID-19) Pandemic. J Am Coll Cardiol. 2020.

${ }^{7}$ Razai MS, Doerholt K, Ladhani S, Oakeshott P. Coronavirus disease 2019 (covid-19): A guide for UK GPS. Vol. 368, The BMJ. BMJ Publishing Group; 2020. p. m800.

${ }^{8}$ Daugherty Biddison EL, Faden R, Gwon HS, Mareiniss DP, Regenberg AC, Schoch-Spana M, et al. Too Many Patients... A Framework to Guide Statewide Allocation of Scarce Mechanical Ventilation During Disasters. Vol. 155, Chest. Elsevier Inc; 2019. p. 848-54.

${ }^{9}$ Palacio Lapuente J. Covid-19 y Atención Primaria. Las oleadas que vienen: Altas hospitalarias, pacientes críticos sin tratamiento hospitalario y atención demorada que pasa a ser indemorable o urgente - semFYC [Internet]. Sociedad Española de Medicina de Familia y Comunidad (SEMFyC); [citado 9 de abril de 2020]. Available at: https://www.semfyc.es/covid-19-y-atencion-primaria-las-oleadas-que-vienen-altashospitalarias-pacientes-criticos-sin-tratamiento-hospitalario-y-atencion-demorada-que-pasa-a-serindemorable-o-urgente/

${ }^{10}$ de Lusignan S, Lopez Bernal J, Zambon M, Akinyemi O, Amirthalingam G, Andrews N, et al. Emergence of a Novel Coronavirus (COVID-19): A Protocol for Extending Surveillance Used by the Royal College of General Practitioners (RCGP) Research and Surveillance Centre (RSC) and Public Health England (PHE) (Preprint). JMIR Public Heal Surveill. 7 de março de 2020;6(2):e18606.

${ }^{11}$ Haines A, de Barros EF, Berlin A, Heymann DL, Harris MJ. National UK programme of community health workers for COVID-19 response. Lancet. março de 2020;

${ }^{12}$ Croda J, Oliveira WK de, Frutuoso RL, Mandetta LH, Baia-da-Silva DC, Brito-Sousa JD, et al. COVID-19 in Brazil: advantages of a socialized unified health system and preparation to contain cases - Rede APS [Internet]. Revista da Sociedade Brasileira de Medicina Tropical. 2020 [citado 9 de abril de 2020]. p. e20200167. Available at: https://redeaps.org.br/2020/04/08/covid-19-in-brazil-advantages-of-asocialized-unified-health-system-and-preparation-to-contain-cases/

${ }^{13}$ Minué S, Massuda A, Tasca R. Debate virtual discute estratégias para a APS durante a pandemia de Covid-19. Rede APS; 2020.

14 Vitória ÂM, Campos GW de S. Só com APS forte o sistema pode ser capaz de achatar a curva de crescimento da pandemia e garantir suficiência de leitos UTI - frente estamira de caps [Internet]. Frente Estamira de CAPS. 2020 [citado 9 de abril de 2020]. Available at: https://frenteestamira.org/2020/04/02/so-com-aps-forte-o-sistema-pode-ser-capaz-de-achatar-acurva-de-crescimento-da-pandemia-e-garantir-suficiencia-de-leitos-uti/ 
ISSN 2596-3317 - DOI 10.14295/aps.v2i1.68

Nedel, F. B.

15 Giovanella L, Rizzotto MLF. Atenção Primária à Saúde: da Declaração de Alma Ata à Carta de Astana. Saúde em Debate. setembro de 2018;42(spe1):6-8.

16 Giovanella L, Rizzotto MLF. Atenção Primária à Saúde: da Declaração de Alma Ata à Carta de Astana. Saúde em Debate. setembro de 2018;42(spe1):6-8. 